\title{
Candidatus Dirofilaria Hongkongensis Infections in Humans During 2005 to 2020, in Kerala, India
}

\author{
Anil Kumar, ${ }^{1 \star}$ Ani Sreedhar, ${ }^{2}$ Lalitha Biswas, ${ }^{3}$ Sarga Prabhat, ${ }^{3}$ Parasmal Suresh, ${ }^{3}$ Anisha Asokan, ${ }^{1}$ Rita Mary Tomy, ${ }^{2}$ Vivek Vinod, ${ }^{3}$ \\ Bindu Lakshmanan, ${ }^{4}$ Ajit Nambiar, ${ }^{5}$ and Raja Biswas ${ }^{3}$ \\ ${ }^{1}$ Department of Microbiology, Amrita Institute of Medical Sciences \& Research Centre, Amrita Vishwa Vidyapeetham, Ponekara, Kochi, Kerala, \\ India; ${ }^{2}$ Department of Ophthalmology, Little Flower Hospital and Research Centre, Angamaly, Kerala, India; ${ }^{3}$ Center for Nanosciences and \\ Molecular Medicine, Amrita Vishwa Vidyapeetham, Ponekara, Kochi, Kerala, India; ${ }^{4}$ Department of Veterinary Parasitology, College of Veterinary \& \\ Animal Sciences, Mannuthy, Thrissur, Kerala, India; ${ }^{5}$ Department of Pathology, Amrita Institute of Medical Sciences and Research Center, Amrita \\ Vishwa Vidyapeetham, Ponekkara, Kochi, Kerala, India
}

\begin{abstract}
We tried to determine the epidemiology and species of human dirofilariasis observed at two tertiary care hospitals in Kerala. We searched the hospital database to identify cases of dirofilariosis from January 2005 to March 2020. Along with human isolates, one dog Dirofilaria isolate was also subjected to PCR and sequencing of pan filarial primers cytochrome oxidase subunits 1 and 12S rDNA. We documented 78 cases of human dirofilariosis. The orbit, eyelid, and conjunctiva were the most commonly affected sites. Molecular characterization identified one dog and five human isolates as Candidatus Dirofilaria Hongkongensis. A rare case of subconjunctival infestation by $B$. malayi was also documented. Human dirofilariosis is a public health problem in the state of Kerala in India, and it is mostly caused by Candidatus Dirofilaria Hongkongensis. We propose that all diroifilaria isolates are subjected to sequencing for identification.
\end{abstract}

\section{INTRODUCTION}

Human dirofilariosis is caused by two species of zoonotic filarial nematodes that naturally infect dogs, cats, and wild animals and are transmitted by mosquito species that belong to the Culicidae family. ${ }^{1,2}$ Humans are accidental hosts where the parasite meets a dead end because it is not usually able to attain sexual maturity. ${ }^{1-3}$ The infection is characterized by the presence of pre-adult worms and, rarely, microfilaraemia. ${ }^{2,3}$ Dirofilaria repens and Dirofilaria immitis are of canine origin and are the most common species that cause human infections. ${ }^{1,2}$ Human infections are increasingly being reported, mostly in the form of subcutaneous nodules, and commonly involve the eyelids or conjunctiva. ${ }^{2,4}$ However, the involvement of the thigh, chest, male genitalia, breast, cheek, abdomen, and upper arm has also been reported. ${ }^{2,4}$ Most reports of $D$. repens infections are from Europe and Asia, but D. immitis is more prevalent in North America and Southern Europe. ${ }^{1,2}$ In southern Europe, canine D. immitis infections are highly prevalent. ${ }^{1,2}$ The first cases of human ocular (1975) and subcutaneous (1999) dirofilariosis caused by $D$. repens in India were reported in the state of Kerala. ${ }^{5}$ Since then, cases of $D$. repens infection have been reported in eight other Indian states, and all were identified based on morphological features like the presence of longitudinal ridges on the cuticle. ${ }^{4,5}$ Studies of microfilaremia in dogs in Kerala have reported incidence rates of $13.4 \%$ in Calicut, $24.2 \%$ in Thrissur, and $7.59 \%$ in Ernakulam districts. ${ }^{5}$ In 2012, a new species, Candidatus Dirofilaria Honkongenesis, was proposed as a causative agent for dirofilariosis in dogs and humans. ${ }^{6} \mathrm{~A}$ recent study performed in Kerala has also identified Candidatus Dirofilaria Honkongenesis in humans and dogs. ${ }^{7}$ We describe the clinical and molecular characteristics

* Address correspondence to Anil Kumar, Amrita Institute of Medical Sciences \& Research Centre, Amrita Vishwa Vidyapeetham, Ponekkara, Kochi, Kerala, India 682041. E-mail: vanilkumar@aims.amrita. edu of human dirofilariosis observed at two tertiary care centers in Kerala.

\section{METHODS}

This study was approved by the institutional review board of both participating hospitals (IRB-AIMS-2018$176 ; 2018 / 89 /$ IEC/LF). We searched the hospital database to identify cases of dirofilariosis from January 2005 to March 2020. Cases from 2005 to 2017 were identified based on morphological worm features in histopathological sections. From 2018 to 2020 , PCR and sequencing were used to identify the parasite in clinical specimens using pan filarial primers targeting cytochrome oxidase subunit 1 (COX1) (Figure 1A) and 12S rDNA (Figure 1B), as described previously. ${ }^{8}$ All COX1 PCR-positive samples were subjected to $12 \mathrm{~S}$ rDNA-based multiplex PCR that could simultaneously identify $D$. repens and $D$. immitis (Figure 1C). ${ }^{9}$ Seven random human isolates and one dog Dirofilaria isolate were sequenced and analyzed using the NCBI BLASTn program. The 12S rRNA and COX1 sequences, along with the sequences retrieved from the GenBank, were subjected to the phylogenetic analysis. Ascaris lumbricoides was used as the outgroup for phylogenetic analysis. The evolutionary history was inferred by using the Maximum Likelihood method and Kimura 2parameter model (Mega X Version 10.1.8). The accuracy of the tree topology was assessed using 100 bootstrap replications. ${ }^{10}$ Scanning electron microscopy (SEM) examinations of the surgically removed worm were performed by fixing $1-\mathrm{cm}$ sections of the worm in $2.5 \%$ glutaraldehyde using JEOL JSM 6490LA microscope.

\section{RESULTS}

During the 15-year period, we identified 78 cases of dirofilariosis. The median age was 45.5 years (9 months-81 years); $24.35 \%(N=19)$ of patients were of pediatric age (younger than 14 years). The youngest patient was 9 months old and presented with a nodule on the dorsum of 
A

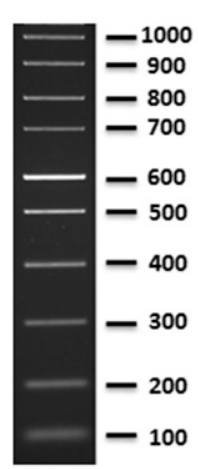

B

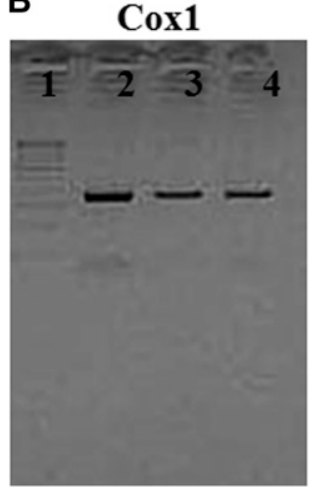

C

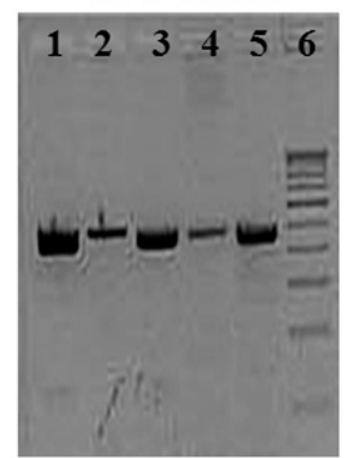

D Multiplex

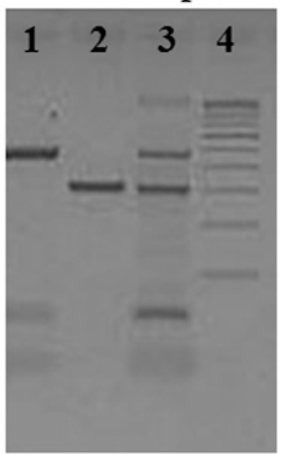

FiguRE 1. (A) A 100-bp DNA ladder. (B) PCR products of the Cox1 gene: lane 1, 100-bp DNA ladder; lane 2, DNA from canine (DNA from dog heartworm); lanes 3 and 4, DNA from human eye specimens. The 321-bp PCR products seen in lanes 2, 3, and 4 indicate the presence of Dirofilaria sp. (C) PCR product of 12S rDNA gene: lane 1, PCR-amplified product from dog heartworm DNA; lanes 2 to 5 , PCR-amplified product from roundworm DNA obtained from a human eye; lane 6, 100-bp DNA ladder. The 506-bp PCR products seen in lanes 1 to 5 indicate the presence of Dirofilaria sp. (D) Multiplex PCR analysis: lane 1, PCR-amplified product using 12S rDNA forward and $12 S$ rDNA reverse primer pairs; lane 2, PCR-amplified product using $12 S$ rDNA forward and $D$. repens reverse primer; lane 3 , multiplex PCR showed a PCR-amplified product using 12S rDNA forward, $12 S$ rDNA reverse, $D$. repens reverse, and $D$. immitis forward primers; lane 4, 100-bp DNA ladder. The 506-bp length fragment in lane 1 represents the Dirofilaria genome. The 327-bp length fragment in lane 2 represents the Dirofilaria repens. The 506-bp genome-specific band and 327-bp species-specific band are seen in lane 3.

the penis. The most commonly affected sites were the eyelid/conjunctiva/orbit $(N=47) ; 59.6 \%$ of cases involved the right side. The second commonest sites were the head/ neck/face $(N=11)$, followed by the breast $(N=6)$, lower limb $(N=3)$, upper limb $(N=2)$, abdomen $(N=2)$, axilla $(N=2)$, and scrotum $(N=2)$. Solitary cases involving the penis, supraclavicular region, and paraspinal regions were also observed. Of the 30 cases with available eosinophil counts, 11 had eosinophilia (> 5\%). The worm was extracted from 20 cases and in the remaining biopsy specimens $(N=58)$ were evaluated to determine the diagnosis. Only 38 specimens collected between 2018 and 2020 were available for PCR; the remainder were morphologically identified as Dirofilaria spp. or D. repens. Sequencing of COX1 and 12S rRNA sequences identified five human isolates (Accession number: MT808309, MT800754, and MT800755) and one dog isolate (Accession number: MT808310) as Candidatus Dirofilaria Honkongenesis. However, the multiplex PCR of one slender live worm extracted from the eye of a 1-year-old girl provided a PCR product only for the 12S rDNA PCR that was negative for species-specific PCR and had a sequence that showed $98.65 \%$ similarity with Brugia malayi (Accession number: MT808311). The COX1 and 12S rRNA sequences of the Dirofilaria species obtained from human and dog isolates were found to be highly conserved. Although the COX1 sequences were $100 \%$ identical, the sequences of 12S rRNA were $99.71 \%$ identical with the existing sequences of the Dirofilaria sp. 'hongkongensis' in the NCBI database. The phylogenetic analysis of COX1 and 12S rRNA sequences of Dirofilaria species also showed that the Candidatus Dirofilaria Honkongenesis clinical isolates from humans and dogs belong to the same clade and are separated from the other Dirofilaria species, $D$. immitits, $D$. repens, and D. ursi (Figure 2). The outgroup Ascaris lumbricoides was clearly separated from the clade for both 12S rDNA and COX1 sequences of the Dirofilaria species. The SEM images of the Candidatus Dirofilaria Honkongenesis surface at different magnifications showed the longitudinal cuticular ridges, which were similar to D. repens (Figure 3).

\section{DISCUSSION}

Our study describes the largest series of human dirofilariosis from the state of Kerala and from India. We observed a high (45\%) occurrence of dirofilariosis in active patients (age, 20-49 years). Sequencing of randomly selected isolates in our study identified Candidatus Dirofilaria Honkongenesis in five cases of human dirofilariosis and one case of dog dirofilariosis. We believe that the remaining 33 PCR-positive cases would be identified by sequencing as Candidatus Dirofilaria Honkongenesis. Because morphological features like cuticular ridges are observed in both species, the 40 cases that were morphologically identified as $D$. repens were also likely to be Candidatus Dirofilaria Honkongenesis. $D$. repens can be differentiated from Candidatus Dirofilaria Honkongenesis only by sequencing. A recent study by Pradeep et al. identified Candidatus Dirofilaria Honkongenesis in three humans and four dogs in Kerala. ${ }^{7}$ Another sequencing study by Nazar et al. performed in Kerala identified human isolates as $D$. repens, albeit, it was closely related to Candidatus Dirofilaria Honkongenesis. ${ }^{11}$ Therefore, we concluded that human dirofilariasis in the state of Kerala in India is exclusively caused by Candidatus Dirofilaria Hongkongensis, and not by D. repens, as reported by previous studies. Subcutaneous filariasis caused by $B$. malayi has also been noted. Because of its high literacy rate and better access to quality health care in the state of Kerala, more cases are being diagnosing there than in the other states of India. Although the findings of our study may be basic in nature, the data are crucial to stimulating discussions about policy changes and making human dirofilariasis a notifiable disease so that its true burden can be determined and remedial measures can be suggested. Our study had limitations. The generated data are from two tertiary care referral hospitals and do not reflect the true prevalence of human dirofilariasis because most patients may seek treatment at a 

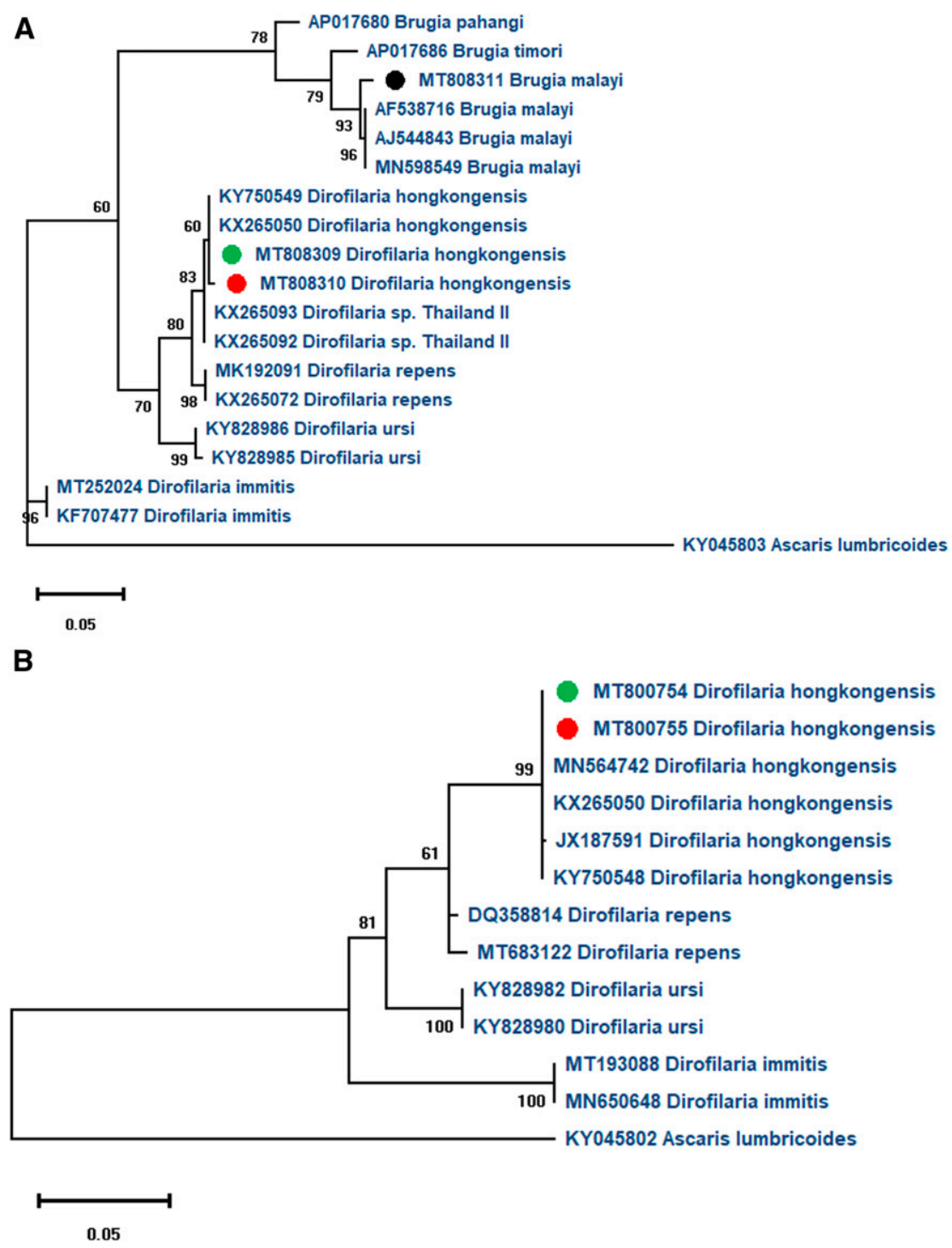

FIGURE 2. Phylogenetic analysis using the Maximum Likelihood method and Kimura 2-parameter model with 1000 bootstrap replications using MEGAX version 10.1.8. (A) Phylogenetic analysis of the 12S rRNA gene sequences of the Dirofilaria species. (B) Phylogenetic analysis of COX1 gene sequences of Dirofilaria species. Ascaris lumbricoides was used as an outgroup for the analysis. Dirofilaria isolates from the present study are indicated by the green dot (human isolate) and red dot (dog isolate). The B. malayi isolate is indicated by the black dot. GenBank accession numbers and species names of the sequences used for the study are shown on the phylogenetic trees. Boot strap values are shown on branches. This figure appears in color at www.ajtmh.org.

local hospital or standalone ophthalmology center. Sequencing of all PCR-positive cases could not be performed because of a paucity of funds. Kerala is endemic to Brugian filariasis, and recent reports have identified dogs as a reservoir host for B. malayi. ${ }^{12}$ Therefore, it was not surprising that one of the worms extracted from the eye of a patient was identified as Brugia malayi.

\section{CONCLUSION}

Dirofilariasis in the state of Kerala in India is exclusively caused by Candidatus Dirofilaria Hongkongensis. It is not caused by $D$. repens. We propose that all diroifilaria isolates should subjected to PCR and sequencing for identification. Efforts should also concentrate on eliminating the reservoir of infection in the canine population by active case detection and treatment. The vector density needs to be controlled to achieve effective elimination. The increasing trend of human dirofilariosis is worrisome. Without intervention, it may become a significant public health problem.

Received November 30, 2020. Accepted for publication February 11, 2021.

Published online April 12, 2021.

Acknowledgments: We acknowledge the support provided by Dr. C. Gopi Mohan, who analyzed the sequences. The American Society of Tropical Medicine and Hygiene (ASTMH) assisted with publication expenses.

Financial support: This research received no specific grant from any funding source.

Disclosure: No conflict of interest declared.

Authors' addresses: Anil Kumar, Lalitha Biswas, Sarga Prabhat, Parasmal Suresh, Anisha Asokan, Vivek Vinod, Ajit Nambiar, and Raja Biswas, Amrita Institute of Medical Sciences and Research Centre, 


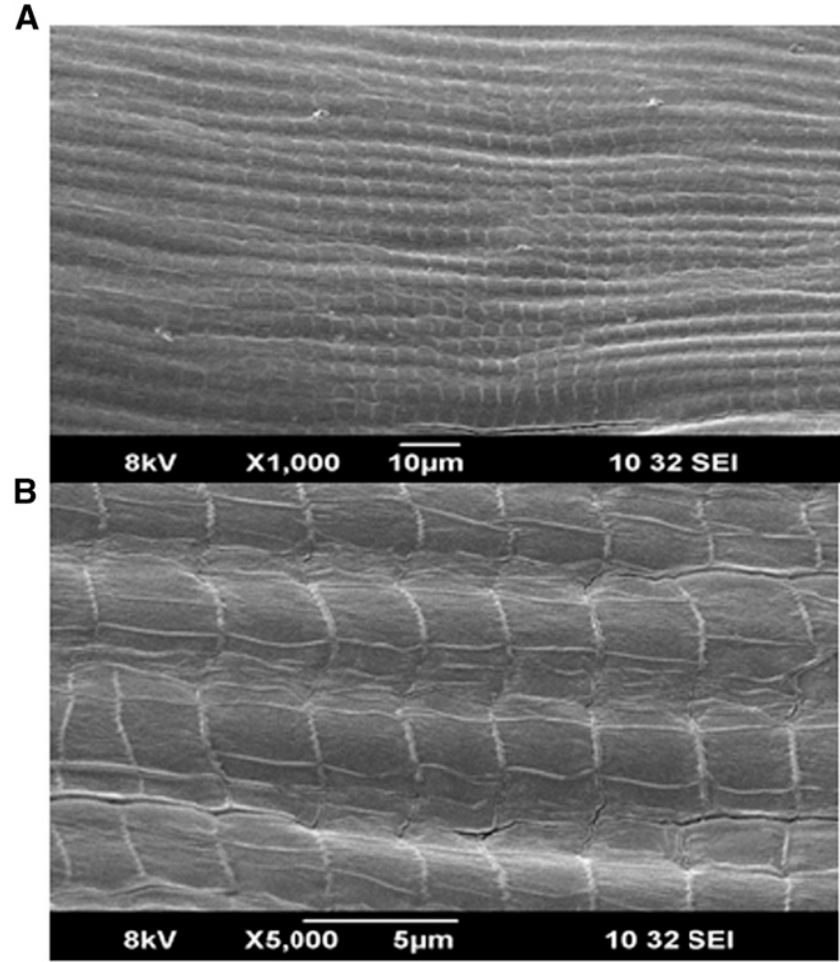

FIGURE 3. Scanning electron microscopy images of the surface of Dirofilaria sp. hongkongensis worm showing longitudinal cuticular ridges in $250 \times$ and $500 \times$ magnifications, respectively.

Molecular Biology, Cochin, Kerala, India, E-mails: vanilkumar@aims. amrita.edu, biswaslalitha@gmail.com, microbiology@aims.amrita.edu, parasmals@aims.amrita.edu, anishaka@gmail.com, vivekvinod@aims. amrita.edu, ajitn@aims.amrita.edu, and rajabiswas@aims.amrita. edu. Ani Sreedhar and Rita Mary Tomy, Little Flower Hospital and Research Centre, Ophthalmology, Angamaly, Kerala, India, E-mails: anisreedhar2002@yahoo.com and ritamarytomy@gmail.com. Bindu Lakshmanan, College of Veterinary and Animal Sciences Mannuthy, Veterinary Parasitology, Mannuthy, Kerala, India, E-mail: bindul@ kvasu.ac.in.

\section{REFERENCES}

1. Genchi C, Rinaldi L, Mortarino M, Genchi M, Cringoli G, 2009. Climate and Dirofilaria infection in Europe. Vet Parasitol 163: 286-292.

2. Simón F, Siles-Lucas M, Morchón R, Gonzalez-Miguel J, Mellado I, Carretón E, Montoya-Alonso JA, 2012. Human and animal dirofilariosis: the emergence of a zoonotic mosaic. Clin Microbiol Rev 25: 507-544.

3. Lechner AM, Gastager H, Kern JM, Wagner B, Tappe D, 2020. Case report: successful treatment of a patient with microfilaremic dirofilariasis using doxycycline. Am J Trop Med Hyg 102: 844-846.

4. Kini RG, Leena JB, Shetty P, Lyngdoh RH, Sumanth D, Lovely G, 2015. Human dirofilariosis: an emerging zoonosis in India. J Parasit Dis 39: 349-354.

5. Sabu L, Devada K, Subramanian H, 2005. Dirofilariosis in dogs and humans in Kerala. Indian J Med Res 121: 691-693.

6. To KK et al., 2012. A novel Dirofilaria species causing human and canine infections in Hong Kong. J Clin Microbiol 50: 35343541.

7. Pradeep RK et al., 2019. Whether Dirofilaria repens parasites from South India belong to zoonotic Candidatus Dirofilaria hongkongensis (Dirofilaria sp. hongkongensis)? Infect Genet Evol 67: 121-125.

8. Winkler S, Pollreisz A, Georgopoulos M, Bagò-Horvath Z, Auer H, To KK, Krücken J, Poppert S, Walochnik J, 2017. Candidatus Dirofilaria hongkongensis as causative agent of human ocular filariosis after travel to India. Emerg Infect Dis 23: 1428-1431.

9. Gioia G, Lecová L, Genchi M, Ferri E, Genchi C, Mortarino M, 2010. Highly sensitive multiplex PCR for simultaneous detection and discrimination of Dirofilariai mmitis and Dirofilaria repens in canine peripheral blood. Vet Parasitol 172: 160163.

10. Tamura K, Nei M, 1993. Estimation of the number of nucleotide substitutions in the control region of mitochondrial DNA in humans and chimpanzees. Mol Biol Evol 10: 512-526.

11. Nazar N, Lakshmanan B, Jayavardhanan KK, 2017. Molecular characterization of human Dirofilaria isolates from Kerala. Indian J Med Res 146: 528-533.

12. Sadarama PV, Chirayath D, Pillai UN, Unny NM, Lakshmanan B, Sunanda C, 2019. Canine Brugia malayi microfilarial excretory/ secretory protein-based antibody assay for the diagnosis of Brugian filariasis in dogs. J Parasit Dis 43: 549-553. 